Revue Revue de l'histoire des religions

de Ihistoire des religions temps des Réformes religieuses

\title{
Affaires de foi. Éthiques et pratiques économiques au temps des Réformes religieuses
}

Avant-propos

Marion Deschamp et Eléna Guillemard

\section{OpenEdition \\ Journals}

Édition électronique

URL : http://journals.openedition.org/rhr/10846

DOI : 10.4000/rhr.10846

ISSN : 2105-2573

Éditeur

Armand Colin

\section{Édition imprimée}

Date de publication : 15 décembre 2020

Pagination : $507-510$

ISBN : 978-2-200-93328-9

ISSN : 0035-1423

Référence électronique

Marion Deschamp et Eléna Guillemard, «Affaires de foi. Éthiques et pratiques économiques au temps des Réformes religieuses », Revue de l'histoire des religions [En ligne], 4 | 2020, mis en ligne le 15 décembre 2020, consulté le 15 janvier 2021. URL : http://journals.openedition.org/rhr/10846 ; DOI https://doi.org/10.4000/rhr.10846 


\title{
Affaires de foi Éthiques et pratiques économiques au temps des Réformes religieuses
}

\author{
Avant-propos
}

Les articles réunis dans ce numéro se proposent d'interroger les rapports entre économie et religion à l'époque moderne. Ils s'intéressent plus précisément aux manières dont ceux-ci sont reformulés, réinventés et transformés dans le contexte des Réformes qui marquent la rupture de l'unité religieuse au sein du christianisme occidental et président à l'émergence d'un régime de pluralité confessionnelle. Si la causa Lutheri qui ébranle l'Europe au début du $\mathrm{XVI}^{\mathrm{e}}$ siècle est bien une affaire de foi, l'expérience du schisme religieux révèle aussi à quel point l'affairement des partisans des Réformes religieuses pour restaurer la vraie doctrine contenue dans la Parole de Dieu est en bonne part liée à la réformation des rapports économiques, que ceux-ci aient à faire avec l'économie du salut, ou qu'ils se jouent sur le plan de l'économie mondaine et du commerce entre les hommes.

Lorsque, en effet, Luther publie ses 95 thèses contre le commerce des Indulgences, ce n'est pas seulement la vénalité de Rome et de ses agents que le moine augustin s'emploie à dénoncer. À travers la critique de la marchandisation des biens du salut, c'est toute l'économie traditionnelle de la foi qui se trouve ébranlée. À une vision mercantile et comptable de l'au-delà, Luther oppose 
la gratuité du salut, don accordé par Dieu aux hommes sans contrepartie, en souvenir du sacrifice du Christ. Au-delà de la coïncidence des lexiques utilisés pour décrire ces phénomènes ${ }^{1}$, il semble bien que deux ordres de réalité économiques soient visés par la Réforme initiée par Luther. Sur le plan théologique, l'économie du salut passe du modèle de l'achat à celui du don gratuit. Sur le plan pécuniaire, l'économie de l'Église, c'est-à-dire l'administration de la fortune terrestre de l'institution romaine, se trouve radicalement rejetée.

La critique des dérives financières de l'Église déborde d'ailleurs rapidement vers d'autres sujets de plaintes économiques. La dénonciation du luxe de l'Église et de l'abandon des principes de charité, argument phare des premières années de la Réforme, est rapidement complétée par des arguments économiques d'ordre plus général. Très vite, les réformateurs de première génération prennent position sur l'usure, les prêts à intérêts, les formations commerciales monopolistiques, les recouvrements de dettes, la prise en charge publique des nécessiteux, etc.

Par ailleurs, la Réforme évangélique suscite des bouleversements économiques (sécularisation des biens ecclésiastiques, professionnalisation du clergé, réinvention de l'économie charitable) qui, dans certains lieux et certains contextes, modifient en profondeur les circuits privilégiés de l'échange, les principes d'allocation monétaire ou la valeur des biens distribués au sein des groupes sociaux traditionnels comme des communautés de foi nouvellement constituées. Enfin, elle suscite, à l'échelle individuelle, de nombreuses configurations et trajectoires de vie inédites, articulant, selon une gamme de multiples possibles, conversions religieuses et logiques de reconversions économiques.

En se donnant pour objet d'études les enjeux et défis économiques posés par les «affaires de foi » engendrées par les Réformes religieuses, ce dossier n'entend ainsi sacrifier a priori aucun des aspects liés à la refonte de l'économie de la foi, que

1. Sur l'interpénétration des lexiques théologiques et économiques dans la tradition de pensée occidentale et, notamment, l'usage de raisonner en termes économiques à l'aide du langage de la théologie (et vice et versa), voir avant tout Giacomo Todeschini, Les Marchands et le Temple. La société chrétienne et le cercle vertueux de la richesse du Moyen Âge à l'époque moderne, Paris, Albin Michel, 2017. 
celle-ci s'exprime dans les discours théologiques des réformateurs de première génération (Martin Luther, Jean Calvin, Pierre Viret ou Menno Simons, par exemple) ou qu'elle se révèle par la formalisation de nouvelles conduites et pratiques économiques, procédant de l'adoption d'un nouvel ethos religieux.

Bien sûr, l'héritage wébérien, et notamment l'idée selon laquelle les Réformes protestantes auraient présidé à une différenciation des cultures économiques expérimentées des deux côtés de la frontière confessionnelle et auraient fait naître des dispositions variées à l'avènement de la modernité économique, est bien présent, et discuté, sous des aspects divers, dans plusieurs contributions de ce dossier'. Mais ce dernier n'a pas pour ambition de prolonger, sur le terrain théorique, la riche et complexe discussion, entamée par Weber et poursuivie par de nombreux penseurs après lui, sur les affinités électives entre éthique protestante et esprit capitaliste ou encore sur le rôle de cette rencontre dans le métarécit de la modernité économique. Bien plutôt, il s'agit ici de faire un pas de côté, quitte à se tenir parfois à distance de ce cadre et horizon d'analyse, pour mieux se ressaisir de manière empirique d'un objet d'étude saturé par les constructions théoriques ${ }^{3}$.

La période séminale des $\mathrm{XVI}^{\mathrm{e}}$ et $\mathrm{XVII}^{\mathrm{e}}$ siècles, privilégiée dans les articles proposés, permet par exemple d'explorer la diversité des positions des réformateurs de la première génération sur une économie chrétienne idéale, leurs portées divergentes sur les nouvelles manières de gérer leurs propres affaires conformément à leur confession de foi et la pluralité des expériences et pensées économiques apparues au sein des différentes confessions protestantes (luthériennes, calvinistes, anabaptistes, etc.).

Par ailleurs, plusieurs auteur.e.s ont choisi non pas de s'en tenir au seul monde de la Réforme protestante, mais de privilégier les situations d'entre-deux, celles marquées par les moments de bascule (individuelle, familiale ou collective) d'un espace confessionnel à l'autre, en soulignant à quel point les choix de religion sont à l'origine de situations inédites de décisions, de stratégies ou encore

2. On se réfère ici principalement à Max Weber, L'Éthique protestante et l'esprit du capitalisme, trad. et éd. par Jean-Pierre Grossein, Paris, Gallimard, 2004.

3. Voir sur ce point Marion Deschamp, «Histoire du protestantisme et histoire économique : quel horizon au-delà de Weber?», Chrétiens et sociétés, à paraître en 2020 . 
de contraintes économiques, que celles-ci président aux sorties de couvent des religieuses converties aux idées réformées, aux destins des huguenots contraints à l'exil et à l'abandon, temporaire ou définitif, de leurs moyens de subsistance ou encore aux déploiements des réseaux commerciaux des compagnies marchandes selon des logiques aussi bien financières que confessionnelles.

À la volonté de dépasser, par la variété des cas d'études proposés, l'image d'une réponse homogène de la Réforme protestante aux défis économiques qu'elle motive ou subit, s'ajoute aussi l'ambition de considérer certaines réponses proposées de l'autre côté de la frontière confessionnelle. En effet, pour faire face aux attaques menées par leurs adversaires en religion contre l'économie traditionnelle des dévotions, les catholiques proposent, notamment, des voies renouvelées d'investissement dans le sacré, ouvertes par de nouveaux espaces missionnaires. Cet évasement du champ d'études vers le monde des Réformes catholiques, bien que trop rapidement esquissé ici, pourra poser les bases de futures discussions sur la pertinence du critère confessionnel dans la fabrique de nouvelles économies de la foi et dans la formulation du débat sur le rôle du religieux dans la transformation des rapports économiques de la première modernité.

Ainsi, c'est une histoire «par le bas » des affaires de foi qui est ici privilégiée, avec une attention particulière portée aux acteurs et contextes singuliers, seuls à même de faire émerger à terme de nouveaux questionnaires qui profiteraient des dynamiques de recherche apparues aussi bien dans le champ de l'histoire religieuse que dans celui de l'histoire économique.

marion.deschamp@univ-lorraine.fr elena.guillemard@laposte.net 